\title{
Comparison of glenohumeral joint rotation between asymptomatic subjects and patients with subacromial impingement syndrome using cine-magnetic resonance imaging: a cross-sectional study
}

Tomonori Kenmoku ${ }^{1,2^{*}}$ (D), Keisuke Matsuki ${ }^{2,3}$, Nobuyasu Ochiai ${ }^{4}$, Masaru Sonoda ${ }^{5}$, Takumi Ishida ${ }^{5}$, Shuichi Sasaki ${ }^{6}$, Yuji Tanaka ${ }^{7}$, Mitsufumi Nakawaki ${ }^{1}$, Naoshige Nagura', Ryo Tazawa' ${ }^{1}$, Yu Sasaki ${ }^{8}$, Scott A. Banks ${ }^{2}$ and Masashi Takaso ${ }^{1}$

\begin{abstract}
Background: The purpose of this study to compare glenohumeral joint motion during active shoulder axial rotation between subacromial impingement syndrome (SIS) shoulders and asymptomatic shoulders using cine-magnetic resonance imaging (cine-MRI). Measurement of glenohumeral joint motion via manual intervention does not assess the usual glenohumeral joint motion, and the glenoid surface cannot be confirmed manually. However, cine-MRI can produce clear images of glenohumeral joint rotation. Therefore, we sought to measure the active ROM of the glenohumeral rotation using cine-MRI.

Methods: Seventy-three shoulders in 42 asymptomatic volunteers and 110 SIS shoulders in 103 consecutive patients were included in this study. We evaluated 36 matched pairs (72 shoulders in total) adjusting for baseline characteristics with propensity score matching method. The patients underwent cine-MRI during axial rotation of the adducted arm. During imaging, participants rotated their shoulder from the maximum internal rotation to the maximum external rotation over the first $10 \mathrm{~s}$ and then back to the maximum internal rotation over the subsequent $10 \mathrm{~s}$. We assessed internal/external rotation, and compared the asymptomatic and SIS shoulders in this regard. Evaluation of rotation angles was performed on a series of axial images through the humeral head center.
\end{abstract}

Results: The mean internal rotation angles of the asymptomatic and patient groups were $55^{\circ} \pm 10^{\circ}$ and $41^{\circ} \pm 23^{\circ}$, respectively, $\left(P=.002 ; 95 \%\right.$ Confidence Interval [Cl], 51-58 vs 33-49); the mean external rotation angles were $47^{\circ} \pm 15^{\circ}$ and $21^{\circ} \pm 25^{\circ}$, respectively, $(P<.001 ; \mathrm{Cl}, 42-52$ vs $13-29)$.

Conclusions: Compared to asymptomatic shoulders, SIS shoulders showed significantly restricted glenohumeral rotation as determined by cine-MRI. Our results suggested that the significant limitation of active glenohumeral rotation might be associated with rotator cuff dysfunction.

Keywords: Subacromial impingement syndrome, Cine magnetic resonance imaging, Shoulder rotation, Rotator cuff

* Correspondence: pseudolefty811@yahoo.co.jp

'Department of Orthopedic Surgery, School of Medicine, Kitasato University,

1-15-1 Kitasato, Minami-ku, Sagamihara, Kanagawa 252-0570, Japan

2Department of Mechanical and Aerospace Engineering, University of Florida,

Gainesville, FL, USA

Full list of author information is available at the end of the article

(c) The Author(s). 2019 Open Access This article is distributed under the terms of the Creative Commons Attribution 4.0 International License (http://creativecommons.org/licenses/by/4.0/), which permits unrestricted use, distribution, and reproduction in any medium, provided you give appropriate credit to the original author(s) and the source, provide a link to the Creative Commons license, and indicate if changes were made. The Creative Commons Public Domain Dedication waiver (http://creativecommons.org/publicdomain/zero/1.0/) applies to the data made available in this article, unless otherwise stated. 


\section{Background}

Subacromial impingement syndrome (SIS) is a common shoulder disorder and is associated with functional impairment and disability of the shoulder [1-5]. The activation of rotator cuff muscles is a fundamental contributor to shoulder joint stability and mobility, which is characterized by centering of the humeral head in the glenoid. Dysfunction of the rotator cuff muscles is considered a major cause of SIS, [1-3] although the precise nature of changes in rotator cuff function in SIS has not been elucidated.

Internal/external glenohumeral rotation is important for shoulder function, because of its association with most shoulder movements, such as abduction or forward flexion $[6,7]$. Previous reports have indicated that internal/external glenohumeral rotation is restricted during elevation in shoulders with SIS [8-10]. The humeral head always rotates spontaneously at the beginning of upper extremity elevation, especially from start to $40^{\circ}$ [11]. It has been suggested that rotator cuff exercise with the arm by the side improves SIS symptoms [4, 12, 13]. Active axial shoulder rotation requires coordination between the internal and external rotators of the glenohumeral joint. Therefore, patients with SIS, compared to asymptomatic individuals, may have rotational dysfunction of the glenohumeral joint with the arm adducted. To date, however, no study has evaluated internal/external glenohumeral rotation with the arm adducted. The common clinical measurement of the rotational angle is a combination of the angles of scapulothoracic and sternoclavicular motion [14]. Thus, it is difficult to assess glenohumeral joint rotation accurately.

Cine-magnetic resonance imaging (cine-MRI) allows dynamic evaluation of subjects and has been used in various fields, such as dynamic evaluation of cardiac function $[15,16]$. Although several analyses of shoulder axial rotation with cine-MRI have been documented, [17-19] the recorded motion was not truly dynamic; images of incremental movement were used instead. Recent advances in MRI systems now allow the acquisition of one to two images per second for cine-MRI, allowing the analysis of dynamic joint motion [20]. Particularly, axial MRI images of the shoulder can provide reliable images for measuring the active rotational angle of the glenohumeral joint noninvasively.

The purpose of this study was to use cine-MRI to compare glenohumeral joint motion during active shoulder axial rotation between SIS shoulders and asymptomatic shoulders. We hypothesized that the range of shoulder axial rotation with the arm in an adducted position in patients with SIS was restricted compared to asymptomatic controls.

\section{Methods}

\section{Participants}

The experimental protocol was conducted in accordance with the guidelines of Kitasato University Medical Ethics
Organization for Clinical Research (KMEO B11-87). The Institutional Review Board of our institute approved the protocol for this study, and all participants (or their parents, if they were underage) provided written informed consent.

This study was of a cross-sectional design. Between January 2009 and December 2013, 73 shoulders of 42 asymptomatic volunteers (18 women, 24 men; asymptomatic group) with a mean age of 28 years (range, 2140) were enrolled in this study (Table 1). The patients had no history of shoulder pain or trauma around the shoulder girdle, including the spine. Because we recruited asymptomatic volunteers at two facilities, two physical therapists in each facility confirmed that the shoulder range of motion (ROM) was not limited and that the painful arc sign, [21] Neer impingement sign, [2] and Hawkins-Kennedy impingement sign [22] were all negative. It was also confirmed via MRI that none of the volunteers had glenohumeral osteoarthritis, rotator cuff tears, and increased signal sign in the glenohumeral joint capsule in the T2-weighted coronal, sagittal, and axial images.

Between January 2012 and December 2015, 155 consecutive patients (169 shoulders) with a mean age of 54 years (range, 15-81) suspected of having SIS without global loss of passive ROM $\left(\leq 100^{\circ}\right.$ of forward flexion, $\leq 10^{\circ}$ of external rotation with the arm adducted, and internal rotation of < the L5 level), [23] underwent cine-MRI at one of our two institutes. Before MRI, we excluded patients who experienced pain at rest or/and both active and passive internal and external rotation with the arm in an adducted position, to eliminate the influence of pain on rotational motion $(n=17)$. However, we included patients who felt pain at the end of rotation $(n=40)$. We also excluded patients with external rotation lag sign, which suggested rotator cuff tears $(n=16),[24]$ and patients with collagen diseases $(n=4)$. A single surgeon confirmed that all patients were positive for one of the following tests: painful arc sign, [21] Neer impingement sign, [2] or Hawkins-Kennedy impingement sign [22]. Fifteen patients (18 shoulders) that were diagnosed with partial or full-thickness rotator cuff tears via MRI (T2weighted coronal and sagittal images) were excluded. No one showed increased signal or capsule edema around the axillary recess in fat-suppressed T2-weighted MR images, which suggested capsulitis related to glenohumeral joint motion (Sensitivity, 85.3-88.2\%; Specificity, 88.2\%) [25, 26]. In addition, no one showed severe glenohumeral joint deformity. Thus, 110 shoulders in 103 patients $(70$ men and 33 women; patient group) with a mean age of 50 years (range, 15-81) were included this study (Table 1).

We noted a significant difference in age and gender when the rotational angles were compared between the asymptomatic subjects and patients with SIS. Hence, we generated 1:1 matched groups to facilitate 
Table 1 Demographic data of subjects

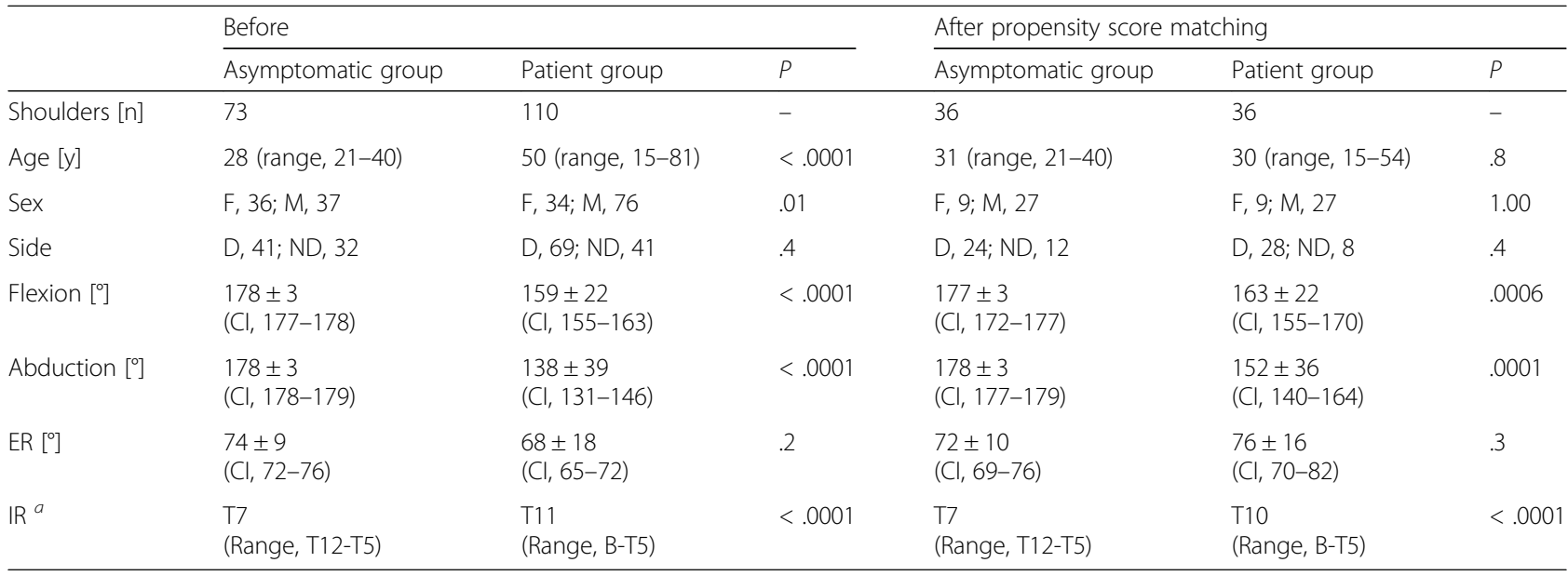

$F$ female, $M$ male, $D$ dominant, $N D$ nondominant, $E R$ external rotation, $I R$ internal rotation, $T$ thoracic vertebra, $B$ buttock, $C I, 95 \%$ confidence interval

${ }^{a} \mathrm{IR}$ was determined by the spine level that could be reached by the thumb

the comparison between the normal subjects and patients on the basis of the propensity score, which was calculated via multivariate logistic regression analysis for each subject, and included confounders for age, sex, and handedness. After matching the study group by the propensity score, a total of 72 shoulders were considered for this investigation comparing the results of ROM determined by cine-MRI between the asymptomatic subjects and patients (Table 1).

\section{Clinical assessment}

One orthopedic surgeon measured the active ROMs for flexion, abduction, and internal and external rotation of the adducted shoulders with the patients seated. Active ROMs of the shoulder were measured following the concept of Cave and Roberts for defining a zero position of the glenohumeral joint with a goniometer [14]. All patients were also evaluated using the Constant score [27] and the UCLA Shoulder Rating Scale (UCLA scale) [28].

\section{MRI acquisition}

Imaging was performed with a $1.5 \mathrm{~T}$ MRI system using a four-channel shoulder array coil (Signa, GE Medical Systems, Milwaukee WI, USA) or with a $1.5 \mathrm{~T}$ system with one of the manufacturer's sized shoulder coils (shoulder 16, Magnetom Aera, Siemens Healthcare, Malvern PA, USA). Cine-MRI of the shoulder was performed using the twodimensional fast imaging employing steady-state acquisition (FIESTA) technique (GE system) (imaging parameters: TR/ $\mathrm{TE}=4.6 / 2.1 \mathrm{msec}$; flip angle, $20^{\circ}$; bandwidth, $\pm 62.5 \mathrm{kHz}$; matrix, $256 \times 224$; number of excitations, 1.0; field of view, $28 \times 28 \mathrm{~cm}$; section thickness, $6.0 \mathrm{~mm}$ ) or using true fast imaging with steady state precession (True FISP) (Siemens Healthcare) (imaging parameters: TR/TE $=4.91 / 2.46 \mathrm{msec}$; flip angle, $20^{\circ}$; bandwidth, $349 \mathrm{~Hz} /$ pixel; matrix, $256 \times 256$; number of excitations, 1.0; field of view, $28 \times 28 \mathrm{~cm}$; section thickness, $6.0 \mathrm{~mm})$. Sequential images were recorded at a rate of 1 image/sec during the activity. Image acquisition was performed on axial slices that included the center of the humeral head, which was determined with a best-fit circle for the humeral head on a scout oblique coronal image.

Subjects performed internal and external rotation of the shoulder in the supine position with the arm adducted. Soft plate cushions were placed under the arm to maintain its long axis parallel to the trunk. Acquisition began with the arm fully rotated internally (with the dorsum of the hand on the greater trochanter). They rotated the arm to the maximum external rotation for over $10 \mathrm{~s}$ and then reversed to the maximum internal rotation for the subsequent $10 \mathrm{~s}$. Before MRI acquisition, all subjects practiced the motion several times following the instructions of a doctor or therapist. We recorded the motion of at least two sets of the activity for each subject.

\section{MRI evaluation}

We assessed the internal/external rotation angles of the patients, and compared them between the asymptomatic controls and SIS shoulders.

Rotation angle was defined as the angle formed by the glenoid and humeral head axes (Fig. 1). The glenoid axis was defined as a line perpendicular to the midpoint of the glenoid fossa. The humeral head axis was defined as a line connecting the midpoint of the articular surface and the center of the humeral head that was the center of the best-fit circle applied to the humeral head. When the two axes were parallel, the joint was considered to be in the neutral position. One examiner, who had 10 years' experience in shoulder research, measured the angle in all subjects. This examiner was blinded to all patient personal and clinical information. Measurement 


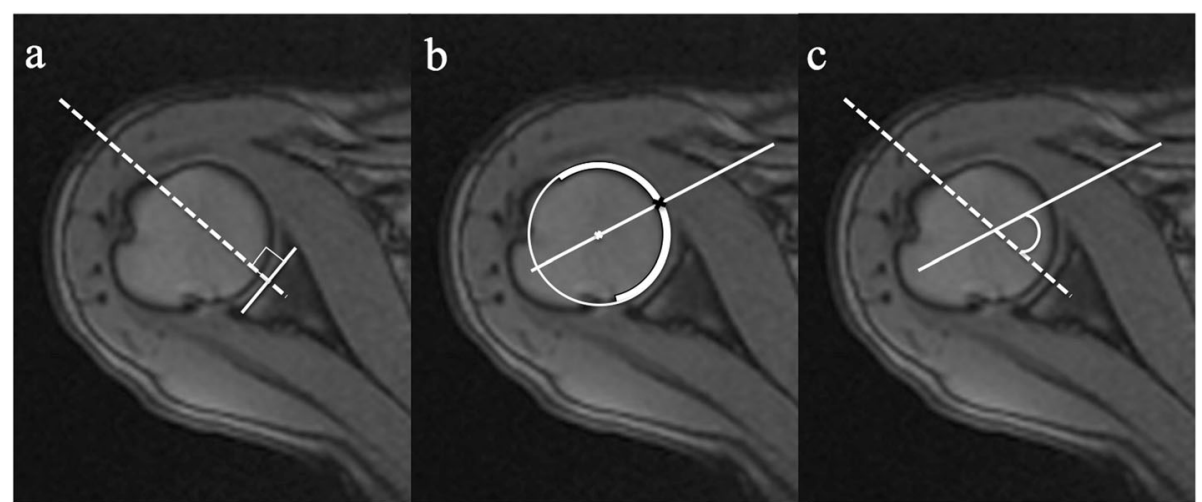

Fig. 1 Rotation angle of the glenohumeral joint. The glenoid axis (dotted line) was defined as a line perpendicular to the surface of the glenoid fossa at its midpoint (a). The humeral head axis (white line) was defined as a line connecting the midpoint of the articular surface (black $\mathbf{x}$ ) to the center of the humeral head (white dot), which was the center of the best-fit circle (white circle) applied to the humeral head (b). The articular surface was identified by the articular cartilage (bold white line). The rotation angle was defined as the angle formed by the glenoid and humeral head axes (c)

was performed on all recorded activities in each subject, and the largest value was used for further assessment. The maximum angle of internal/external rotation from 2 trials was accepted as the rotational angle in each case.

\section{Statistical analysis}

Statistical analysis was performed using commercial software (JMP Pro version 12.2, SAS Institute Inc., Cary NC, USA). Results are presented as the mean \pm standard deviation (SD). The Welch t-test was used to compare quantitative variables. Receiver operating characteristic (ROC) analysis was performed to determine diagnostic cutoff values of rotational angles for SIS. The area under the curve (AUC) was used to assess the accuracy of the analysis, with values $>0.9$ considered highly accurate and 0.7-0.9 moderately accurate [29]. We also compared sex and affected side using chi-square test. For all statistical analyses, significance was set at $P<.05$.

Interclass correlation coefficients (ICC) were calculated to investigate inter- and intra-examiner reliability for the angle measurements. For inter-examiner reliability, two shoulder surgeons with 5 and 10 years' experience in shoulder research, respectively, independently measured 40 randomly selected shoulders, and $\operatorname{ICC}(2,1)$ was determined. For intra-examiner reliability, the surgeon with 10 years' experience measured 40 shoulders twice at a 1-week interval, and ICC $(1,1)$ was determined. All examiners were blinded to the patients' personal and clinical information.

\section{Results}

\section{Participants' demographics}

One shoulder surgeon confirmed that no patient had a progressive global restriction of the shoulder that was related to a severe frozen shoulder [23] at least 1 month after MRI. Comparison of the active ROMs of the asymptomatic controls and the SIS shoulders, measured by the goniometer, revealed significant differences in all directions except external rotation (Table 1). The number of patients who were positive in each clinical test is listed in Table 2. The mean Constant score and UCLA scale of patients after propensity score matching were $73.5 \pm 13.1$ and $19.8 \pm$ 3.4 , respectively. The available data of this study included in the Additional file 2.

Inter- and intra- examiner reliability indicated excellent agreement (ICC $[1,2]=0.99 ; 95 \%$ Confidence interval $[\mathrm{CI}]$, 0.95-0.99; Standard error of mean $[\mathrm{SEM}]=1.13$; Minimal detectable change $[\mathrm{MDC}]_{95}=3.14$; ICC $[1]=0.98 ; \mathrm{CI}$, 0.97-0.99; $\mathrm{SEM}=1.99 ; \mathrm{MDC}_{95}=7.78$ ).

\section{Comparison of cine-MRI data between asymptomatic shoulders and shoulders with SIS}

Cine-MRI could obtain scans of the active glenohumeral rotation with vivid clarity in all asymptomatic shoulders and shoulders with SIS (Figs. 2, 3; an additional movie file shows this in more detail (see

Table 2 The number of patients who were positive in each clinical test

\begin{tabular}{ll}
\hline Clinical test & $\mathrm{n}$ \\
\hline The painful arc sign & 19 \\
Neer sign & 21 \\
Hawkins-Kennedy sign & 28 \\
Painful arc sign and Neer sign & 0 \\
Painful arc sign and Hawkins-Kennedy sign & 4 \\
Neer sign and Hawkins-Kennedy sign & 3 \\
All signs & 13 \\
\hline
\end{tabular}




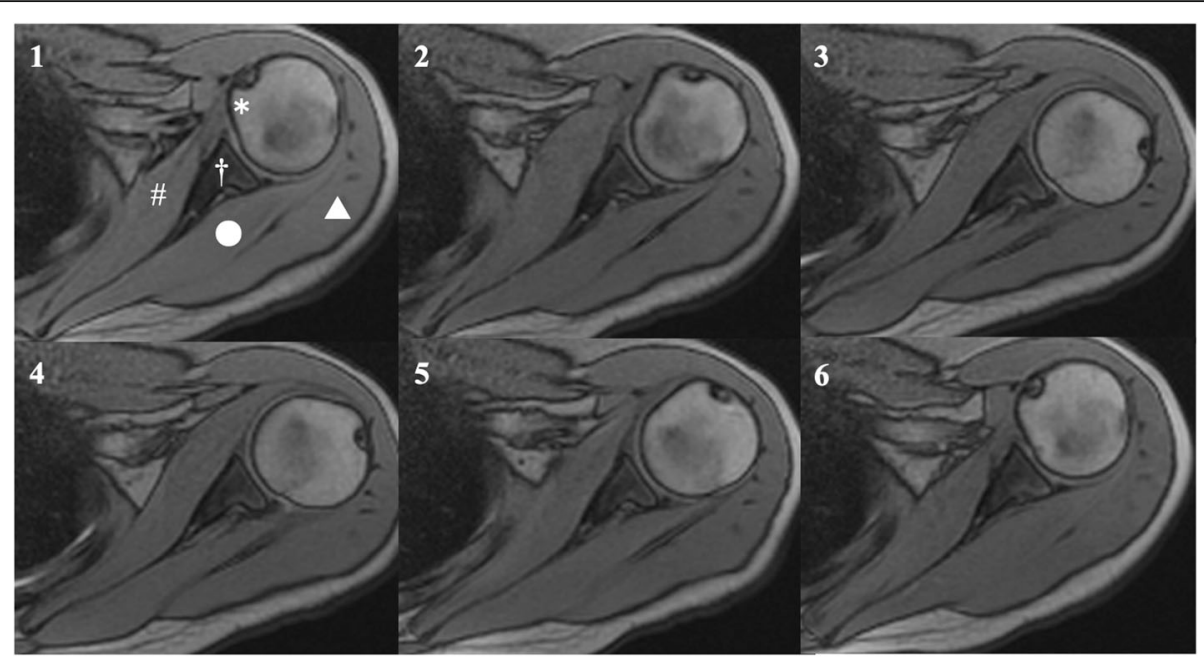

Fig. 2 Motion of an asymptomatic volunteer. The left shoulder of a participant. The movement of the subscapularis and infraspinatus muscles of an asymptomatic shoulder was clearly visible using cine-MRI and no blurring was observed. 1, Starting position; 2, Motion in external rotation; 3, Point at the maximum external rotation; 4 and 5, Motion in internal rotation; 6, End position. *, The lesser tuberosity of the humeral head; \#, The subscapularis; †, Glenoid; $\circ$, The infraspinatus; $\Delta$, The deltoid

Additional file 1)). The mean internal rotation angles of the asymptomatic and patient groups were $55^{\circ} \pm 10^{\circ}$ and $41^{\circ} \pm 23^{\circ}$, respectively, $(P=.002 ; \mathrm{CI}, 51-58$ and 33-49, respectively; Mean difference [MD], - 14; Standard error difference [SED], 4); mean external rotation angles were $47^{\circ} \pm 15^{\circ}$ and $21^{\circ} \pm 25^{\circ}$, respectively, $(P<.001 ; \mathrm{CI}, 42-52$ and $13-29$, respectively; MD, 26; SED, 5). The results of ROC analyses showed the cutoff values of rotation angles in cine-MRI studies with a high diagnostic accuracy (Fig. 4).

\section{Discussion}

Consistent with our hypothesis, glenohumeral internal/external rotation with the arm adducted was significantly restricted in shoulders with SIS compared to the asymptomatic controls. However, there were no significant difference in the active ROM of external rotation measured physically. Our findings may suggest that rotator cuff exercise with the arm adducted improves SIS symptoms [4, 12, 13].

The significant limitation of active glenohumeral rotation determined by cine-MRI may be associated with

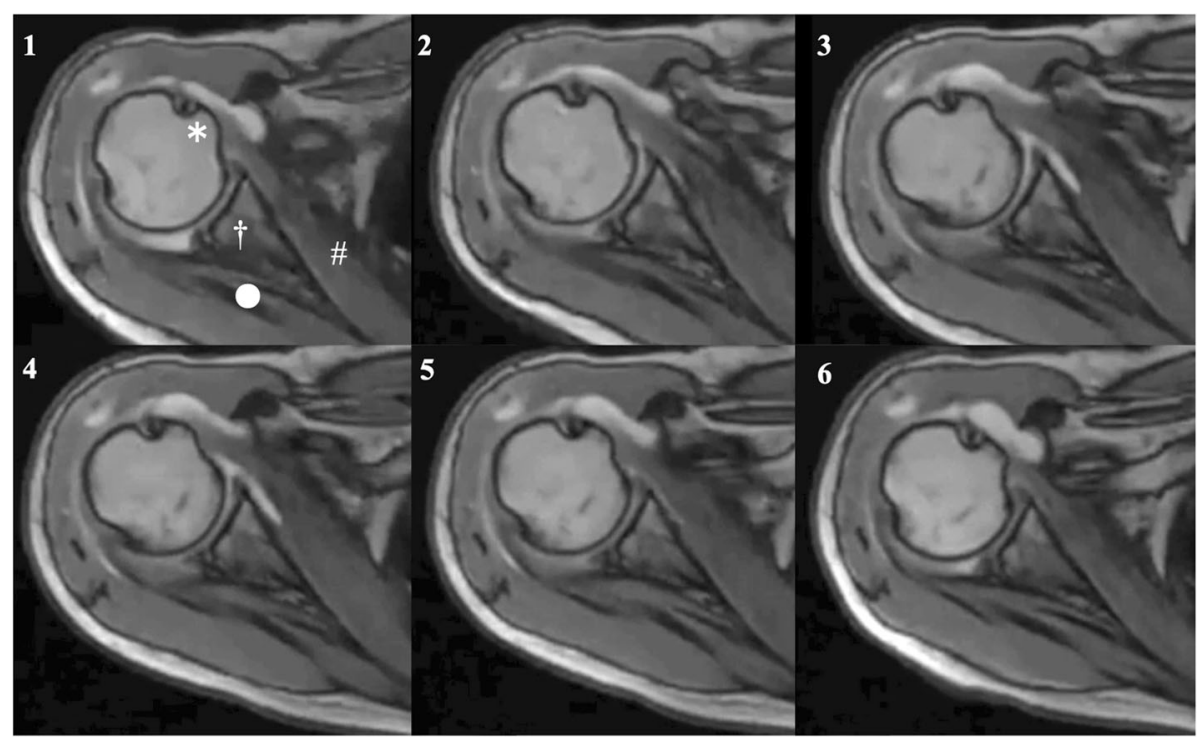

Fig. 3 Limitation in external rotation of shoulder with shoulder impingement syndrome. The right shoulder of a patient with shoulder impingement syndrome. Cine-MRI also produced clear images of the movements of the glenohumeral joint rotation in a shoulder with SIS. 1 , Starting position; 2, Motion in external rotation; 3, Point at the maximum external rotation; 4 and 5, Motion in internal rotation; 6, End position. *, The lesser tuberosity of the humeral head; \#, The subscapularis; $\dagger$, Glenoid; 0 , The infraspinatus 

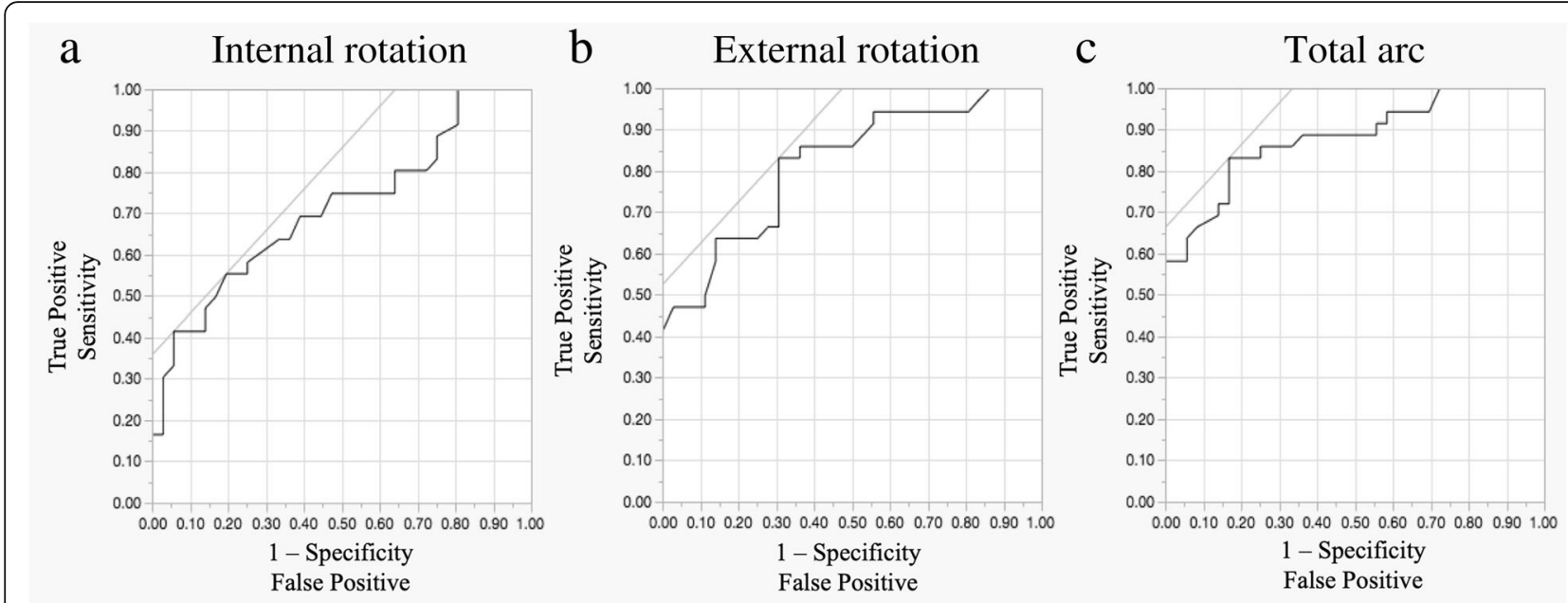

Fig. 4 Receiver operating characteristic (ROC) analysis a, internal rotation; b, external rotation; c, total arc. According to ROC analyses, the cutoff values for diagnosis of shoulder impingement syndrome were $47^{\circ}$ for internal rotation (sensitivity, 0.56 ; specificity, 0.81 ; sensitivity - [1-specificity], 0.36; AUC, 0.71 ), $41^{\circ}$ for external rotation (sensitivity, 0.83 ; specificity, 0.69 ; sensitivity - [1-specificity], 0.53; AUC, 0.82), and $86^{\circ}$ for total arc (sensitivity, 0.83; specificity, 0.83; sensitivity - [1-specificity], 0.67; AUC, 0.88)

rotator cuff dysfunction. The infraspinatus and subscapularis should work as principal external and internal rotators, respectively, during axial rotation of the adducted arm [7, 12, 30, 31]. Imbalance of the rotator cuff muscles is considered one of the causes of SIS, $[3,13,32]$ and previous studies have reported that internal/external rotator activities were significantly lower in shoulders with SIS compared to asymptomatic shoulders [33, 34]. Decreased activity of the infraspinatus and subscapularis may have been related to the limited internal/external rotation in SIS shoulders in this study. Additionally, our study sample included individuals with end-range active ROM pain. A bidirectional relationship exists between end-range pain and active ROM (e.g., pain may limit active ROM, and limited active ROM may induce pain); we could not dissociate this relationship. Further studies are required to clarify the contribution of end-range pain to active ROM in SIS.

Many authors have reported that the limitation of glenohumeral rotation is related to symptoms of SIS $[8,9$, 35]. The findings of these reports are consistent with our results. The pathology of SIS is considered to predispose the shoulder to mechanical compression in the subacromial space due to excessive superior translation of the humeral head during elevation $[2,3,36]$. The infraspinatus and subscapularis work as depressors of the humeral head during elevation $[1,31]$. Therefore, the decreased rotational function of the infraspinatus and subscapularis suggested by our results may also be related to the increased superior translation of the humeral head. Since we did not analyze rotational differences during elevation, further research are necessary to confirm this hypothesis.

Although many shoulders exhibited severe rotational limitation as shown by the MRI measurements, we were unable to detect rotational limitations with the measurement of active ROM of external rotation measured physically. One possible explanation for this discrepancy may be the differences in functional compensation by the body position. Dysfunction of the rotator cuff muscles can be compensated for by the surrounding muscles and scapular motion, and active ROMs are sometimes maintained even in shoulders with cuff muscle palsy [37, 38]. The scapula has a wide range of motion, and its motion can compensate for limitations in glenohumeral rotation in the standing or sitting position [39]. Contrarily, zero point determined by cine-MRI was the point the axes of the humeral head and glenoid were parallel; therefore, we could measure the glenohumeral rotational angle without compensation. In addition, subjects rotated their arm with the elbow extended in the supine position during MRI acquisition in this study. As this position may have restricted scapular motion, we could isolate glenohumeral rotation. Therefore, the difference in external rotational angle between the two methods may be related to whether the compensation around the shoulder should be excluded or not.

Although cine-MRI is diagnostically informative, it is not feasible in most clinical settings and may not provide sufficient resolution. However, diagnostic ultrasound may be a suitable method for quantifying glenohumeral joint angles. Although current ultrasound-based visualization methods are unable to fully quantify rotator cuff coordination, 
particularly for muscles on the posterior side of the scapula, recent diagnostic and therapeutic advances may make this more feasible in the future [40-42]. Therefore, the cutoff values identified in the present manuscript (Fig. 2) may provide a suitable reference point for diagnostic ultrasoundbased assessments of SIS and post-therapeutic gains in the future.

\section{Limitations}

The major limitation of this study was its cross-sectional design. A longitudinal study that would examine changes in rotational angles before and after treatment, to assess relationships between symptoms and the rotational angle, may be required. Second, we did not assess muscle activity in this study. Electromyography (EMG) may be required to confirm decreased function of the rotator cuff muscles; however, it is impossible to record EMG and MRI simultaneously. Therefore, the discussion and conclusions of our results as they relate to alterations in muscle activity are theoretic. Third, we could not completely rule out the potential influences of end-range pain or early stage adhesive capsulitis on glenohumeral rotation, although we excluded patients with pain at rest or/and during rotational motion during the simulation exercise before cine-MRI was performed. Therefore, our findings might also include the influence of a pain-related subliminal limitation during rotation. Fourth, we did not exclude the involvement of systemic diseases that may contribute to abnormal motion, although we eliminated apparent inhibiting factors except rotator cuff dysfunction, such as rotator cuff tears, stiff shoulder, or osteoarthritis. Fifth, we did not include individuals who were unable to perform the necessary shoulder rotation within the gantry of the MRI. Therefore, these findings cannot be generalized to obese individuals with SIS. Sixth, the ROC curves were constructed based on a relatively small sample. Considering that cine-MRI has not been reported in this population, these findings can be used as a reference point for future experimentation with cine-MRI as well as with other emerging imaging methods that may improve the clinical diagnosis of SIS.

\section{Conclusions}

We compared glenohumeral rotational motion between asymptomatic control and SIS shoulders using cine-MRI. Compared to asymptomatic shoulders, SIS shoulders showed significantly restricted glenohumeral rotation. However, no significant differences in active ROMs of external rotation measured with a goniometer were found between the asymptomatic and SIS shoulders. The significant limitation of active glenohumeral joint rotation in this study may be associated with rotator cuff dysfunction.

\section{Supplementary information}

Supplementary information accompanies this paper at https://doi.org/10. 1186/s12891-019-2818-3.

Additional file 2. Available data of this study. This excel file included the data of asymptomatic subjects and patients after propensity score matching.

\section{Abbreviations \\ AUC: Area under the curve; Cl: Confidence interval; Cine-MRI: Cine-magnetic resonance imaging; ER: External rotation; FIESTA: Fast imaging employing steady-state acquisition; HSD: Honestly significant difference; ICC: Interclass correlation coefficients; IR: Internal rotation; MD: Mean difference; MDC: Minimal detectable change; ROC: Receiver operating characteristic; ROM: Range of motion; SD: Standard deviation; SED: Standard error difference; SEM: Standard error of mean; SIS: Subacromial impingement syndrome; True FISP: True fast imaging with steady state precession; UCLA scale: The University of California, Los Angeles Shoulder Rating Scale}

\section{Acknowledgments}

The authors thank Shohei Minami, Toshiaki Kotani (Division of Orthopedic Surgery, Seirei Sakura Citizen's Hospital) for administrative support. We also thank Yusuke Inoue (Division of Radiology, Kitasato University School of Medicine), Shogo Nishiyama, and Harumi Watanabe (Division of Radiology, Fuchinobe General Hospital) for administrative and technical support with the cine-MRI.

\section{Authors' contributions}

TK is responsible for the integrity of this study, and he conducted the enrollment of patients with SIS. KM provided logistic support, interpreted cine-MRI data, and revised the manuscript. MS, TI, and YS provided imaging of cine-MRI and were involved in the writing of the Methods section. SS and YT enrolled the healthy volunteers and measured their shoulder ROM, and were involved in the writing of the Methods and Results sections. MN measured the cine-MRI data and was involved in the writing of the Introduction and Results sections, while SAB provided logistic support for statistical analyses and revised the Methods, Results, and Discussion sections. $\mathrm{NO}, \mathrm{NN}$, and RT managed the subjects, deciding the condition of cine-MRI, and analyzed the data. MT provided administrative and logistic support during the study, and contributed to data interpretation and critical revision of this manuscript. All authors approved the final version of the manuscript.

\section{Funding}

TK was supported by JSPS KAKENHI [Grant no. 25861340]. The funding body had no control over the design of the study; the collection, analysis, and interpretation of data, or the writing of this manuscript.

Availability of data and materials

We have uploaded the dataset we used for this study as an excel file [Available data of propensity].

Ethics approval and consent to participate

All participants (or their parents, if they were underage) provided written informed consent. The Institutional Review Board of our institute (Kitasato University Medical Ethics Organization for Clinical Research; KMEO B11-87) approved the protocol for this study.

Consent for publication

All patients gave written informed consent for this publication.

\section{Competing interests}

The authors have no conflicts of interest to declare.

\section{Author details}

${ }^{1}$ Department of Orthopedic Surgery, School of Medicine, Kitasato University, 1-15-1 Kitasato, Minami-ku, Sagamihara, Kanagawa 252-0570, Japan. ${ }^{2}$ Department of Mechanical and Aerospace Engineering, University of Florida, Gainesville, FL, USA. ${ }^{3}$ Funabashi Orthopaedic Sports Medicine \& Joint Center, Funabashi, Japan. ${ }^{4}$ Department of Orthopedic Surgery, Chiba University Graduate School of Medicine, Chiba, Japan. ${ }^{5}$ Division of Radiology, Seirei 
Sakura Citizen's Hospital, Sakura, Japan. ${ }^{6}$ Division of Rehabilitation, Kitasato University East Hospital, Sagamihara, Japan. 'Division of Rehabilitation, Seire Sakura Citizen's Hospital, Sakura, Japan. ${ }^{8}$ Division of Orthopaedic Surgery, Seirei Sakura Citizen's Hospital, Sakura, Japan.

\section{Received: 9 September 2018 Accepted: 6 September 2019} Published online: 25 October 2019

\section{References}

1. Sharkey N, Marder R. The rotator cuff opposes superior translation of the humeral head. Am J Sports Med. 1995;23:270-5.

2. Neer CS 2nd. Anterior acromioplasty for the chronic impingement syndrome in the shoulder: a preliminary report. J Bone Joint Surg Am. 1972;54:41-50.

3. Michener LA, McClure PW, Karduna AR. Anatomical and biomechanical mechanisms of subacromial impingement syndrome. Clin Biomech. 2003;18:369-79.

4. Jobe FW, Moynes DR. Delineation of diagnostic criteria and a rehabilitation program for rotator cuff injuries. Am J Sport Med. 1982;10:336-9.

5. Chang K, Mezian K, Nanka O, Wu W, Lin W, Özçakar L. Ultrasound-guided interventions for painful shoulder: from anatomy to evidence. J Pain Res. 2018;11:2311-22.

6. Anglin C, Wyss UP, Pichora DR. Glenohumeral contact forces. Proc Inst Mech Eng H. 2000;214:637-44.

7. Kuechle DK, Newman SR, Itoi E, Niebur GL, Morrey BF, An KN. The relevance of the moment arm of shoulder muscles with respect to axial rotation of the glenohumeral joint in four positions. Clin Biomech. 2000;15:322-9.

8. Hallström E, Kärrholm J. Shoulder kinematics in 25 patients with impingement and 12 controls. Clin Orthop Relat Res. 2006;448:22-7.

9. Ludewig PM, Cook TM. Alterations in shoulder kinematics and associated muscle activity in people with symptoms of shoulder impingement. Phys Ther. 2000;80:276-91.

10. Poppen NK, Walker PS. Normal and abnormal motion of the shoulder. J Bone Joint Surg Am. 1976;58:195-201.

11. Ludewig PM, Phadke V, Braman JP, Hassett DR, Cieminski CJ, LaPrade RF. Motion of the shoulder complex during multiplanar humeral elevation. J Bone Joint Surg Am. 2009;91:378-89.

12. Reinold MM, Wilk KE, Fleisig GS, Zheng N, Barrentine SW, Chmielewski T, et al. Electromyographic analysis of the rotator cuff and deltoid musculature during common shoulder external rotation exercises. J Orthop Sports Phys Ther. 2004;34:385-94.

13. Morrison DS, Frogameni AD, Woodworth P. Non-operative treatment of subacromial impingement syndrome. J Bone Joint Surg Am. 1997;79:732-7.

14. Cave E, Roberts S. A method for measuring and recording joint function. J Bone Joint Surg Am. 1936;18:455-65.

15. Barkhausen J, Ruehm SG, Goyen M, Buck T, Laub G, Debatin JF. MR evaluation of ventricular function: true fast imaging with steady-state precession versus fast low-angle shot cine MR imaging: feasibility study. Radiology. 2001;219:264-9.

16. Heitner J, Bhumireddy GP, Crowley AL, Weinsaft J, Haq SA, Klem I, et al. Clinical application of cine-MRI in the visual assessment of mitral regurgitation compared to echocardiography and cardiac catheterization. PLoS One. 2012;7:e40491.

17. Friedman RJ, Bonutti PM, Genez B. Cine magnetic resonance imaging of the subcoracoid region. Orthopedics. 1998;21:545-8.

18. Allmann KH, Uhl M, Gufler H, Biebow N, Hauer MP, Kotter E, et al. Cine-MR imaging of the shoulder. Acta Radiol. 1997;38:1043-6.

19. Bonutti PM, Norfray JF, Friedman RJ, Genez BM. Kinematic MRI of the shoulder. J Comput Assist Tomogr. 1993;17:666-9.

20. Kenmoku T, Miyajima G, Ochiai N, Yamazaki H, Sasaki Y, Yamaguchi T, et al. The comparison between active and passive rotation of shoulder joint using cine-MRI. Katakansetsu. 2014;38:403-6.

21. Kessel L, Watson M. The painful arc syndrome. Clinical classification as a guide to management. J Bone Joint Surg Br. 1977;59:166-72.

22. Hawkins RJ, Kennedy JC. Impingement syndrome in athletes. Am J Sports Med. 1979;8:151-8

23. Ueda Y, Sugaya H, Takahashi N, Matsuki K, Kawai N, Tokai M, et al. Rotator cuff lesions in patients with stiff shoulders: a prospective analysis of 379 shoulders. J Bone Joint Surg Am. 2015;97:1233-7.

24. Hertel R, Ballmer FT, Lombert SM, Gerber C. Lag signs in the diagnosis of rotator cuff rupture. J Should Elbow Surg. 1996;5:307-13.
25. Park S, Lee DH, Yoon SH, Lee HY, Kwack KS. Evaluation of adhesive capsulitis of the shoulder with fat-suppressed T2-weighted MRI: association between clinical features and MRI findings. Am J Roentgenol. 2016;207:135-41.

26. Gondim Teixeira PA, Balaj C, Chanson A, Lecoca S, Louis M, Blum A. Adhesive capsulitis of the shoulder: value of inferior glenohumeral ligament signal changes on t2-weighted fat-saturated images. Am J Roentgenol. 2012;198:W589-96.

27. Constant $\mathrm{CR}$, Murley $\mathrm{AH}$. A clinical method of functional assessment of the shoulder. Clin Orthop Relat Res. 1987;214:160-4.

28. Ellman $H$, Hanker $G$, Bayer M. Repair of the rotator cuff. End-result study of factors influencing reconstruction. J Bone Joint Surg Am. 1986;68:1136-44.

29. Swets JA. Measuring the accuracy of diagnostic systems. Science. 1988;240:1285-93.

30. Dark A, Ginn KA, Halaki M. Shoulder muscle recruitment patterns during commonly used rotator cuff exercises: an electromyographic study. Phys Ther. 2007;87:1039-46.

31. Otis JC, Jiang CC, Wickiewicz TL, Peterson MG, Warren RF, Santner TJ. Changes in the moment arms of the rotator cuff and deltoid muscles with abduction and rotation. J Bone Joint Surg Am. 1994;76:667-76.

32. Conroy DE, Hayes KW. The effect of joint mobilization as a component of comprehensive treatment for primary shoulder impingement syndrome. J Orthop Sports Phys Ther. 1998:28:3-14.

33. Clisby EF, Bitter NL, Sandow MJ, Jones MA, Magarey ME, Jaberzadeh S Relative contributions of the infraspinatus and deltoid during external rotation in patients with symptomatic subacromial impingement. J Should Elbow Surg. 2008;17(Suppl 1):87S-92S.

34. Reddy AS, Mohr KJ, Pink MM, Jobe FW. Electromyographic analysis of the deltoid and rotator cuff muscles in persons with subacromial impingement. J Should Elbow Surg. 2000;9:519-23.

35. Matsen FA, Arntz CT. Subacromial impingement. In: Rockwood CAJ, Matsen FA, Arntz CT, editors. . Philadelphia: W.B.Saunders; 1990. p. 623-46.

36. Bigliani LU, York N, Levine WN. Current concepts review - subacromial impingement syndrome. J Bone Joint Surg. 1997;79:1854-68.

37. Chen AL, Ong BC, Rose DJ. Arthroscopic management of spinoglenoid cysts associated with slap lesions and suprascapular neuropathy. Arthroscopy. 2003;19:E15-21.

38. lanotti JP, Ramsey ML. Arthroscopic decompression of a ganglion cyst causing suprascapular nerve compression. Arthroscopy. 1996;12:739-45.

39. Veeger HEJ, van der Helm FCT. Shoulder function: the perfect compromise between mobility and stability. J Biomech. 2007;40:2119-29.

40. Chang KV, Wu WT, Huang KC, Jan WH, Han DS. Limb muscle quality and quantity in elderly adults with dynapenia but not sarcopenia: an ultrasound imaging study. Exp Gerontol. 2018;108:54-61.

41. Chang KV, Wu WT, Han DS, Özçakar L. Static and dynamic shoulder imaging to predict initial effectiveness and recurrence after ultrasound-guided subacromial corticosteroid injections. Arch Phys Med Rehabil. 2017:98:1984-94.

42. Wu WT, Chang KV, Mezian K, Naňka O, Lin CP, Özçakar L. Basis of shoulder nerve entrapment syndrome: an ultrasonographic study exploring factors influencing cross-sectional area of the suprascapular nerve. Front Neurol. 2018;9:1-11.

\section{Publisher's Note}

Springer Nature remains neutral with regard to jurisdictional claims in published maps and institutional affiliations.

Ready to submit your research? Choose BMC and benefit from:

- fast, convenient online submission

- thorough peer review by experienced researchers in your field

- rapid publication on acceptance

- support for research data, including large and complex data types

- gold Open Access which fosters wider collaboration and increased citations

- maximum visibility for your research: over $100 \mathrm{M}$ website views per year

At $\mathrm{BMC}$, research is always in progress.

Learn more biomedcentral.com/submissions 